\title{
Automated identification of Lauraceae by scale-invariant feature transform
}

\author{
Sung-Wook Hwang ${ }^{1} \cdot$ Kayoko Kobayashi $^{1}$ - Shengcheng Zhai ${ }^{2} \cdot J^{\text {Junji Sugiyama }}{ }^{1,2}$ (])
}

Received: 29 September 2017 / Accepted: 16 November 2017 / Published online: 12 December 2017

(C) The Author(s) 2017. This article is an open access publication

\begin{abstract}
An image dataset of the cross-sectional optical micrographs of the Lauraceae species including 39 species in 11 genera, capturing at least one full annual ring, was investigated by scale-invariant feature transform (SIFT), a computer vision-based feature extraction algorithm. We found an image of $900 \times 900$-pixel size at a pixel resolution of ca. $3 \mu \mathrm{m}$, corresponding to the actual size of $2.65 \times 2.65 \mathrm{~mm}^{2}$, as the minimum requirement for the image dataset in terms of the accuracy of the recognition at both the genus and species levels. Among the several classifiers investigated, the linear discriminant analysis (LDA) presented the best performance reaching a maximum of $89.4 \%$ in the genus with a species identification of approximately $96.3 \%$. Cluster analysis of all the SIFT descriptors for each image yielded practical information regarding the descriptors; they recognize selectively the cell lumina, cell corners, vessels, and axial and ray parenchyma cells. Therefore, the difference between the genus or species levels was determined per the variation in the quantities of these computer-based properties. Another clustering approach, the hierarchal dendrogram, was applied to visualize the numerical distances between the genus and species. Interestingly, even Machilus and Phoebe, which are difficult to distinguish by conventional visual inspection, are quite distantly classified at the genus level. In contrast, some species in Cinnamomum, Machilus and Litsea were categorized into different subgroups rather than the original genus. Microscopic wood identification is found to be possible at the genus level; however, the numerical dataset of the morphological features has various overlapping clusters, causing the genus-level identification of the Lauraceae to be more difficult than species-level identification.
\end{abstract}

Keywords Classification $\cdot$ Computer vision $\cdot$ Hierarchical clustering $\cdot$ Linear discriminant analysis $\cdot$ Optical micrograph

\section{Introduction}

The Lauraceae family is distributed worldwide mainly in warm temperate and tropical regions. It is also found in East Asia, and is regarded as an important wood species. For example, in ancient times, Phoebe was used as a coffin material in China, and Cinnamomum camphora was used as a material for building ships and sculptures in Japan. Thus, the Lauraceae are a culturally and historically important family in East Asia. Although the taxonomy of the Lauraceae is not entirely understood, they are known to consist of approximately 45 genera and over 2800 species. The Lauraceae are

Junji Sugiyama

sugiyama.junji.6m@kyoto-u.ac.jp

1 Research Institute for Sustainable Humanosphere, Kyoto University, Gokasho, Uji, Kyoto 611-0011, Japan

2 College of Materials Science and Engineering, Nanjing Forestry University, Nanjing 210037, China known to be relatively difficult to distinguish because of their vast diversity and wide variety within the family [1]. Therefore, the classification of the Lauraceae is complex and not easy. However, an unambiguous classification is essential for understanding their cross-cultural utilization.

Identifying wood species accurately is very important in ethnobotanical research on wood usage. Among the several methods proposed until now, the microscopic method is the most accurate and widely used owing to the tremendous efforts by the wood anatomists and the corresponding identification keys proposed by the IAWA (the international association of wood anatomists) community. The method allows us to mostly identify it up to the genus level.

More recently, identification methods are gradually expanding to new techniques such as DNA-based identification [2-6] and spectroscopy-based chemometrics [7-12]. These methods are used primarily for specific purposes. DNA-based identification is a method that uses the inherent DNA sequence of each species, and has the advantage 
of being able to identify up to the species level. However, owing to the lack of a database, it is difficult to use it for generalized identification. Chemometrics, combining the spectroscopic data and multivariate analysis, is representative of the facile and nondestructive analysis. However, this application is restricted to limited or selected species identification, and its accuracy is negatively affected by aging that is critical when this method is applied to old wood used in cultural properties [9].

Recently, wood identification using computer visionbased methods to extract features from the wood images has been receiving an increasing interest. Several methods such as the gray level co-occurrence matrix (GLCM) [13, 14], local binary pattern (LBP) $[15,16]$, and higher order local autocorrelation (HLAC) [17] have been tested to extract features from wood images. Computer vision is a part of the diverse field of artificial intelligence, and is one of the technologies with the most potential. Currently, computer vision algorithms that can be applied to various problems are being developed by computer scientists. The selection and implementation of these algorithms will vary according to the problem that is considered. For instance, it is known that Haar-like features and histogram of gradient (HOG) exhibit good performance for face recognition and human detection and its motions, respectively $[18,19]$. Scale-invariant feature transform (SIFT) is one of the useful algorithms to detect the local features from an image. It was first introduced by D.G. Lowe in 1999 [20], and was discussed in an extended paper in 2004 [21]. The features detected by SIFT are invariant to scale and rotation, and are robust to the changes in the illumination and affine. The usefulness of SIFT has already been demonstrated in various studies [22-24], and advanced algorithms such as the speeded-up robust features (SURF) [25], principal components analysis-based local descriptor (PCA-SIFT) [26], and gradient location and orientation histogram (GLOH) [24] are being developed starting from SIFT, for inspiring the various descriptors proposed later.

In this study, a wood classification system based on the SIFT algorithm was designed to investigate the Lauraceae family, i.e., the structural and anatomical diversity in the genera and species is systematically and statistically quantified by computer vision. The relationship between the observed computer-based features and anatomical characteristics of wood was comparatively discussed, and the applicability of computer-based features for automated classification was presented. In addition, a hierarchical clustering was performed to analyze the correlations as well as the distances from the genera and species. This paper describes a new approach for the quantification of wood structure and its application to the anatomy that, we believe, can be applied to numerous other fields in wood science.

\section{Experimental procedure}

\section{Image dataset}

The species in the Lauraceae family that are used for wood classification are summarized in Table 1 . The wood samples of 11 genera including 39 species of the Lauraceae were received from the RISH Xylarium, Kyoto University. The cross-section of the samples were cut using a sliding microtome, and stained with safranin. The images were captured at a low magnification with Olympus ${ }^{\mathrm{TM}} 2 \times(0.08 \mathrm{NA})$ PlanApo objective lens, using a BX51 optical microscope equipped with DP73 CCD (charge-coupled device) camera (Olympus, Japan). Most of the acquired images have an area of at least one annual ring. The original size of an image was $4800 \times 3600$ pixels, and the resolution was $0.74 \mu \mathrm{m} /$ pixel.
Table 1 List of the species in the image dataset

\begin{tabular}{|c|c|}
\hline Genus & Species $^{\mathrm{a}}$ \\
\hline Actinodaphne & acutivena $(1)$, forrestii $(1)$, mushaensis (1) \\
\hline Beilschmiedia & linocieroides $(1)$ \\
\hline Cinnamomum & $\begin{array}{l}\text { camphora }(21), \text { glandliferum }(2), \text { japonicum }(15), \text { longipetiolatum }(2), \text { micranthum }(1) \text {, } \\
\text { porrectum }(1), \text { teniplum }(1)\end{array}$ \\
\hline $\begin{array}{l}\text { Cryptocarya } \\
\text { Laurus }\end{array}$ & $\begin{array}{l}\text { chinensis }(1) \\
\text { nobilis }(3)\end{array}$ \\
\hline Lindera & communis (1), glauca (3), thomsonii (2), umbellata (10) \\
\hline Litsea & coreana (18), cubeba (1), elongata (1), glutinosa (2) \\
\hline Machilus & $\begin{array}{l}\text { ichangensis (2), japonica (5), kusanoi (1), pauhoi (2), pingii (2), thunbergii (21), } \\
\text { viridis (2), zuihoensis (1) }\end{array}$ \\
\hline Neolitsea & acuminatissima (1), acutotrinervia (1), cambodiana $(1)$ \\
\hline Phoebe & lanceolata (1), macrocarpa (1), nапти (2), neurantha (2), puwenensis (1), sheareri (2) \\
\hline Sassafras & tzити (3) \\
\hline
\end{tabular}

${ }^{\text {a }}$ The numbers in parenthesis indicate the number of wood blocks from which the image dataset was collected 
The Lauraceae dataset consisted of 1557 images, and each species had more than 8 images.

\section{Image pretreatment}

For feature extraction, all the images in the dataset were converted from RGB color to 8-bit grayscale. An initial dataset of square $3600 \times 3600$-pixel images of $0.74 \mu \mathrm{m} /$ pixel resolution was cropped from the original micrographs, corresponding to an actual area of $2.65 \times 2.65 \mathrm{~mm}^{2}$. Then, a series of datasets with decreasing resolutions, 1.47, 2.94, 5.88, and $11.76 \mu \mathrm{m} /$ pixel were prepared by bilateral filtering. These images of $3600 \times 3600$ pixels to $1800 \times 1800,900 \times 900$, $450 \times 450$, and $225 \times 225$ pixels in the same area were tested for determining the necessary size and resolution information for the classification.

\section{Classification}

The flowchart of the experimental procedure including the image pretreatment is shown in Fig. 1. The image features were calculated from a series of downsized image datasets, followed by classification using multiple classifiers.

\section{SIFT algorithm}

SIFT is selected as the algorithm to extract image features that are known to be invariant to the image scale and rotation. A feature detected by SIFT is called a keypoint. The algorithm generates a difference of Gaussian (DOG) from the image pyramid, and it detects the local extremes as the keypoint candidates in the DOG. Subsequently, the algorithm determines the keypoints that are normally inappropriate as features, by eliminating the candidates present on the edge or having a low-contrast.

In the calculation, we adopted the default parameters proposed by Lowe [21], namely, the number of layers in each octave (nOctaveLayers $=3$ ), value of the Gaussian filter applied to the image of each layer $(\sigma=1.6)$, contrast threshold $(\mathrm{ct}=0.06)$, and edge threshold $(\mathrm{et}=10)$.

\section{Classifiers}

Four different classifiers, namely, k-nearest neighbor (k-NN), logistic regression (Logistic_R), support vector machine (SVM) with a linear kernel, and linear discriminant analysis (LDA) were used for the classification, and the performances of the classifiers were compared. The test set and training set were randomly selected in a ratio of 2:9, and the calculation was repeated 20 times with different sets of test and training data to obtain the average and standard deviation of the accuracy of recognition.

\section{$k$-means clustering}

To visualize the objects that SIFT selects as keypoints, $k$-means clustering was performed on all the detected features from one image of Cinnamomum camphora, and eight representative centroid patterns of the SIFT descriptors were calculated. The keypoints derived from each pattern were reprojected on the corresponding image, and their distribution was scrutinized in comparison with the anatomical features.
Fig. 1 Experimental flowchart of image processing, machine learning, and classification

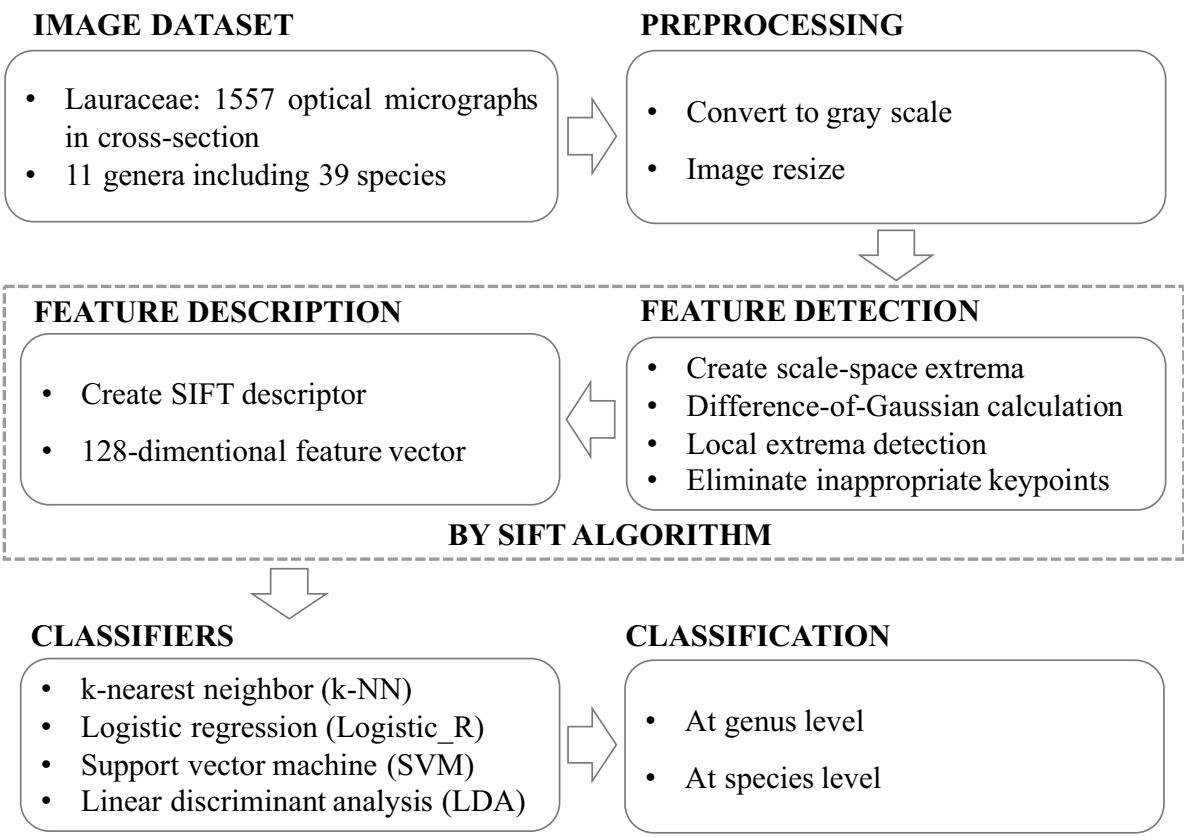




\section{Hierarchical clustering}

An unsupervised hierarchical clustering was performed to draw a dendrogram based on the numerical distances between the genus and species. The simple Euclidean distances calculated from the original SIFT descriptors did not cause a reasonable clustering; therefore, the dimensionality of the descriptors was reduced by LDA. The clustering was performed by the agglomerative Ward's minimum variance method [27] using LDA components up to ten as a dataset. The above setting was selected by considering the cophenetic correlation coefficient [28] that was proposed as a measure of how closely a dendrogram preserved the pairwise distances between the original unclusterized data points.

\section{Programming}

All the programs for the image pretreatments and classification were written in Python 3.5.2 [29] running on MacOSX. The SIFT algorithm and image pretreatments were implemented by the libraries OpenCV [30], NumPy [31], and SciPy [32]. The $k$-means clustering and hierarchical clustering were implemented by the Scikit-learn [33] and SciPy libraries, respectively.

\section{Results and discussion}

\section{SIFT descriptor and classification}

An example of the keypoints detected by the SIFT operation is presented in Fig. 2a. This algorithm determines various local gradient-based keypoints that are expressed by a 128-bins histogram representing the orientation and magnitude of the gradient around them, as shown in Fig. 2b. Numerous keypoints are found from an image. Generally, the descriptor obtained by SIFT and used for further analyses is a single 128-dimensional vector that is in fact the sum of the histograms from all the keypoints in each image (Fig. 2c). Briefly, all the images have their own single SIFT descriptor, and they have only 128-bins to represent themselves.

A summary of the accuracies in the classification of the Lauraceae dataset at various image resolutions is presented in Table 2. Among the four classifiers tested, LDA and SVM yield better results that are depicted in Fig. 3. Clearly, in both the cases, a good performance is obtained from the image dataset having a better resolution, with LDA exhibiting a

Table 2 Classification accuracies of the Lauraceae image dataset at the genus and species levels by the SIFT algorithm with the four different classifiers

\begin{tabular}{llllll}
\hline $\begin{array}{l}\text { Image size } \\
\text { (pixels) }\end{array}$ & $\begin{array}{l}\text { Classification } \\
\text { level }\end{array}$ & \multicolumn{4}{l}{ Classification accuracy $(\%)$} \\
\cline { 3 - 6 } & & k-NN & Logistic_R & LDA & SVM \\
\hline $3600 \times 3600$ & Genus & 85.5 & 74.3 & 86.3 & 84.1 \\
& Species & 82.2 & 79.2 & 93.3 & 87.8 \\
\multirow{2}{*}{$1800 \times 1800$} & Genus & 86.5 & 77.0 & 88.2 & 86.6 \\
& Species & 84.3 & 82.6 & 95.5 & 90.1 \\
$900 \times 900$ & Genus & 83.7 & 81.2 & 89.4 & 88.0 \\
& Species & 80.0 & 89.4 & 96.3 & 95.4 \\
$450 \times 450$ & Genus & 84.6 & 75.9 & 76.5 & 82.7 \\
& Species & 82.5 & 83.6 & 88.8 & 89.8 \\
$225 \times 225$ & Genus & 72.2 & 65.5 & 63.5 & 68.7 \\
& Species & 67.2 & 67.5 & 70.4 & 75.8 \\
\hline
\end{tabular}

SIFT scale-invariant feature transform, $k$ - $N N k$-nearest neighbor, Logistic_R logistic regression, $L D A$ linear discriminant analysis, $S V M$ support vector machine
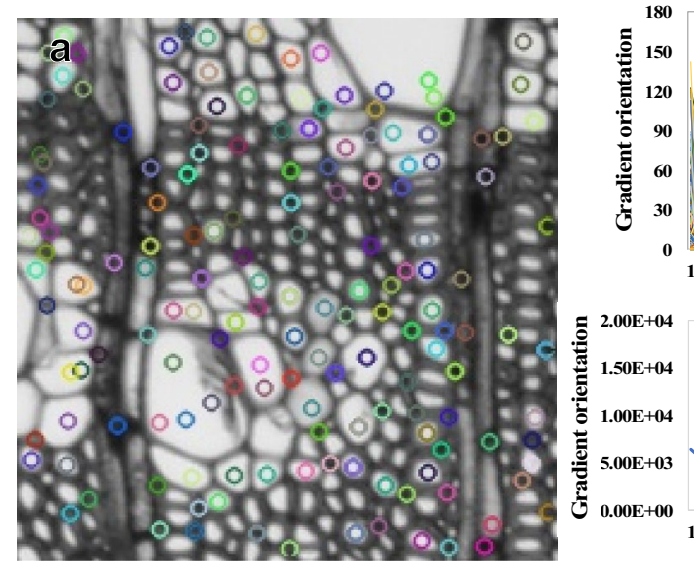

b

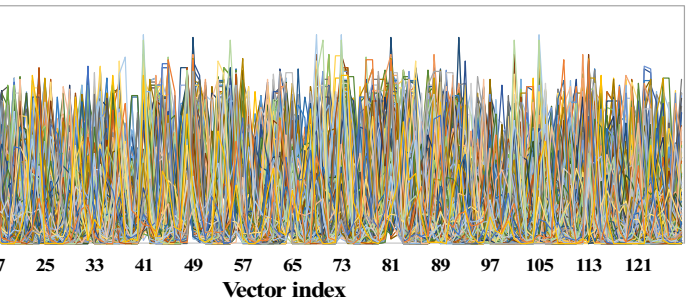

C

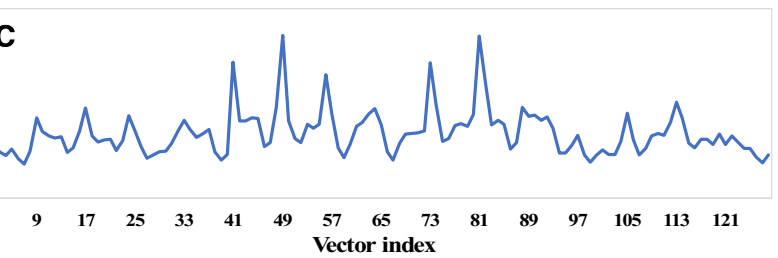

Fig. 2 Keypoints and descriptors in the SIFT algorithm. a Keypoints detected in a cross-section of Cinnamomum camphora. The image is partially cropped to a size of $225 \times 225$ pixels from the original size, and a total of 220 keypoints are detected; $\mathbf{b}$ descriptors of all detected keypoints in the image in $\mathbf{a}$; $\mathbf{c}$ The sum of the SIFT descriptors represented in the image in $\mathbf{a}$ as well as in the histograms in $\mathbf{b}$ 


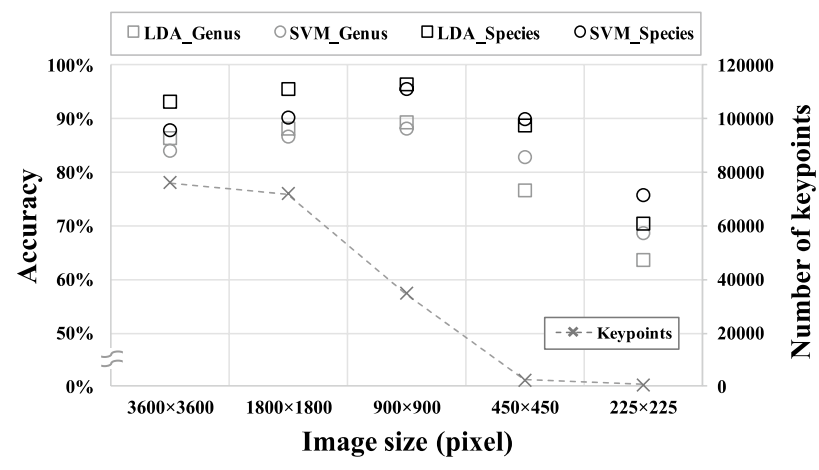

Fig. 3 Variation in the classification accuracy and number of keypoints by reducing the image size at the genus and species levels

slightly better performance than SVM. The best accuracy is $89.4 \%$ by LDA and $88.0 \%$ by SVM in the genus classification, and it is $96.3 \%$ by LDA and $95.4 \%$ by SVM in the species classification mostly, when the $900 \times 900$ image dataset was tested. This implies that the information obtained from the $2.65 \times 2.65 \mathrm{~mm}^{2}$ area with a resolution of $2.94 \mu \mathrm{m}$ possesses the critical information required for identification.

More importantly, it is worth to note that the accuracy of the prediction is better at the species level than the genus level.
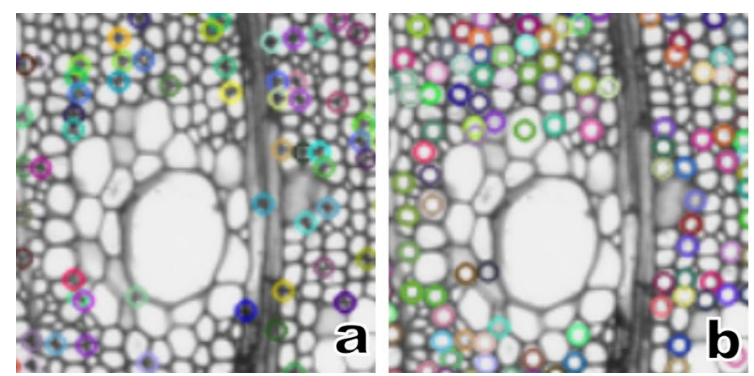

Fig. 4 SIFT keypoints clustered by $k$-means clustering in the crosssection of Cinnamomum camphora. The images show an enlarged part, and the keypoints are detected in an image of $900 \times 900$ pixels.

\section{Structure of SIFT}

To determine the features that can be extracted from the $2.65 \times 2.65 \mathrm{~mm}^{2}$ area with a resolution of $2.94 \mu \mathrm{m}$ in the cross-section of $C$. camphora through computer vision, the SIFT descriptors were examined via cluster analysis. As mentioned earlier, each image provided numerous SIFT descriptors and image-size dependency of the keypoints was plotted as a dashed line in Fig. 3. Obviously, a damping of as much as 5 to 3 digits difference in the number of keypoints occurred in between $900 \times 900$ and $450 \times 450$ pixel images.

All the SIFT descriptors from the image of $900 \times 900$ pixel size were subjected to unsupervised clustering into eight virtual classes, and the keypoints within each class were re-plotted on the initial image. Four representative classes are shown in Fig. 4; the remaining four classes were either similar to one of these presented four classes or were a mix of multiple classes. Expectedly, the keypoints in a given class are found on exactly similar anatomical features, and in this case, they are located at the cell corners, cell lumina, vessel elements, and axial parenchyma cells. The centroid histograms for each class are presented in Fig. 5, showing clear differences in the histogram patterns. In fact, the number of keypoints that belong to these

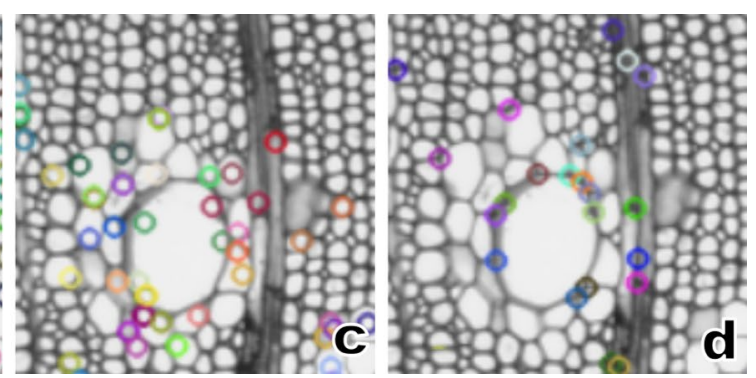

The keypoints in each image are mainly located at the cell corners (a), cell lumina (b), vessel and axial parenchyma cells (c), and cell wall of the vessel and ray parenchyma cells (d)

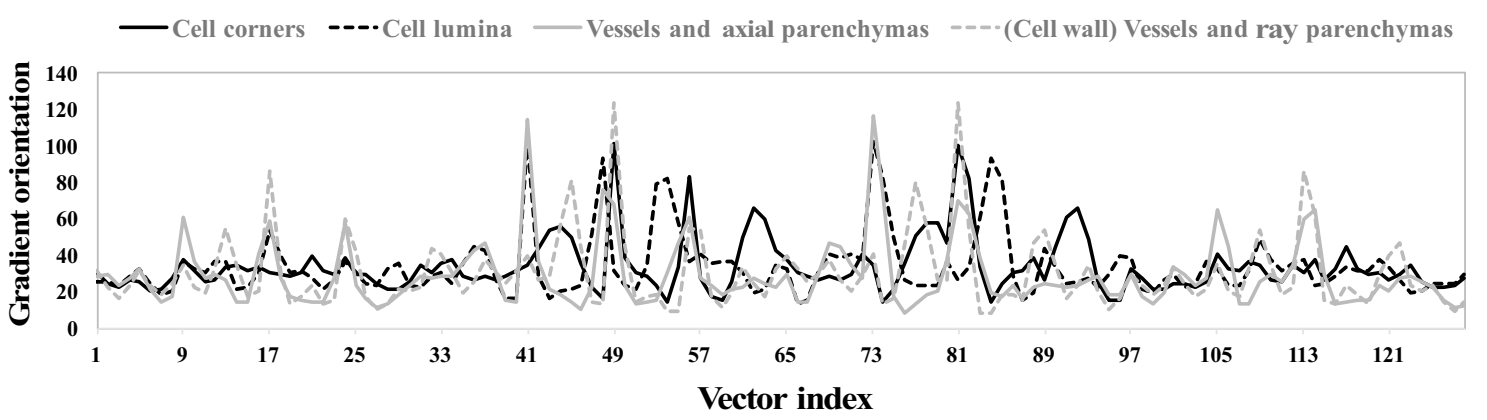

Fig. 5 SIFT descriptors of each class indicating different anatomical features as categorized by $k$-means clustering 
patterns of histograms differs with the image, species, and genus, as a result of which SIFT allows us to perform the classification.

\section{Resolution dependent keypoints distribution}

The $900 \times 900$-pixel images with $2.65 \times 2.65 \mathrm{~mm}^{2}$ area and a resolution of $2.94 \mu \mathrm{m}$ in the cross-section, appear to be the most suitable setting for performing further analysis in terms of the classification accuracy and memory and computational time load. To confirm, the composition of the shift descriptors of the $C$. camphora images was re-investigated with respect to the change in the image resolution. As shown in Fig. 6, the composition of the features is almost equivalent for $3600 \times 3600$ and $1800 \times 1800$-pixel images. In these images, the keypoints detected at the cell corners are approximately $50 \%$ of the total, of which approximately $40 \%$ are detected in the cell lumina. In the case of the $900 \times 900$-pixel image, the compositional ratio at the cell corner and cell lumina decreases to $47.9 \%$ and $36.8 \%$ respectively, which is slightly less than for the larger images. This may be owing to the fact that the feature extraction capability of SIFT is influenced by the image size, and thus, the ratios of the composition of the same anatomical features are somewhat different. However, in comparison with the images smaller than $450 \times 450$ pixels, the $900 \times 900$-pixel image set is found to possess reasonable information that can be provided from the images larger than itself.

Overall, the image size, i.e., the resolution, is a very important factor in the classification, and the $900 \times 900$ pixel images with $2.65 \times 2.65 \mathrm{~mm}^{2}$ area and a resolution of $2.94 \mu \mathrm{m}$ in the cross-section are essential to expect a satisfactory performance. Furthermore, it is interesting to note that computer vision suggested that the numbers or localization of the cell corners are species-specific, and this could never be provided from a human vision.

\section{Unsupervised hierarchical clustering}

Figure 7 shows the dendrograms at the genus and species levels with cophenetic correlation coefficients of 0.60 and 0.65 , respectively. As described in the experimental section, LDA reduced the datasets of the SIFT descriptors for the $900 \times 900$-pixel images. The cophenetic coefficient is the parameter, used in biostatistics, that in the dendrogram closely represents the actual numerical distances between any paired data. Thus, the variation in the data in a given branch is considered to be larger than in the genus compared with in species clustering. This is consistent with the result of the classification accuracy, as described earlier. In genus level clustering shown in Fig. 7a, eleven genera are blanched into 4 groups. Machilus belonged to the same group with Cinnamomum and both the species are in very close distance to each other, although in the actual phylogenetic relationship [5, 6], Machilus is close to Phoebe. In practice, with microscopic inspection, some species in Machilus are known to be difficult to be distinguished from those of Phoebe, even by skilled wood anatomists. As shown in Fig. 7a, in contrast computer vision, Pheobe is sub-grouped differently from the majority of species in Cinnamomum, Machilus and Litsea.

In the dendrogram at the species level (Fig. 7b), various species are sub-grouped independently from their original genus. Specifically, the species in Cinnamomum, Machilus and Litsea have been randomly mixed to form new subgroups. The accuracy of species prediction by LDA being as high as $96 \%$, the dendrograms suggest the presence of morphological similarity, although it is invisible unless computer vision is applied.

\section{Numerical and real phylogenetic trees}

With the classification by SIFT depending only on the crosssectional morphological features of wood, it is not surprising that both at the genus and species levels, the relative
Fig. 6 Variation in the compositional ratio of the anatomical features due to the reduction in the image size. V, vessel; AP, axial parenchyma cell; $R$, ray parenchyma cell; * Standard deviation

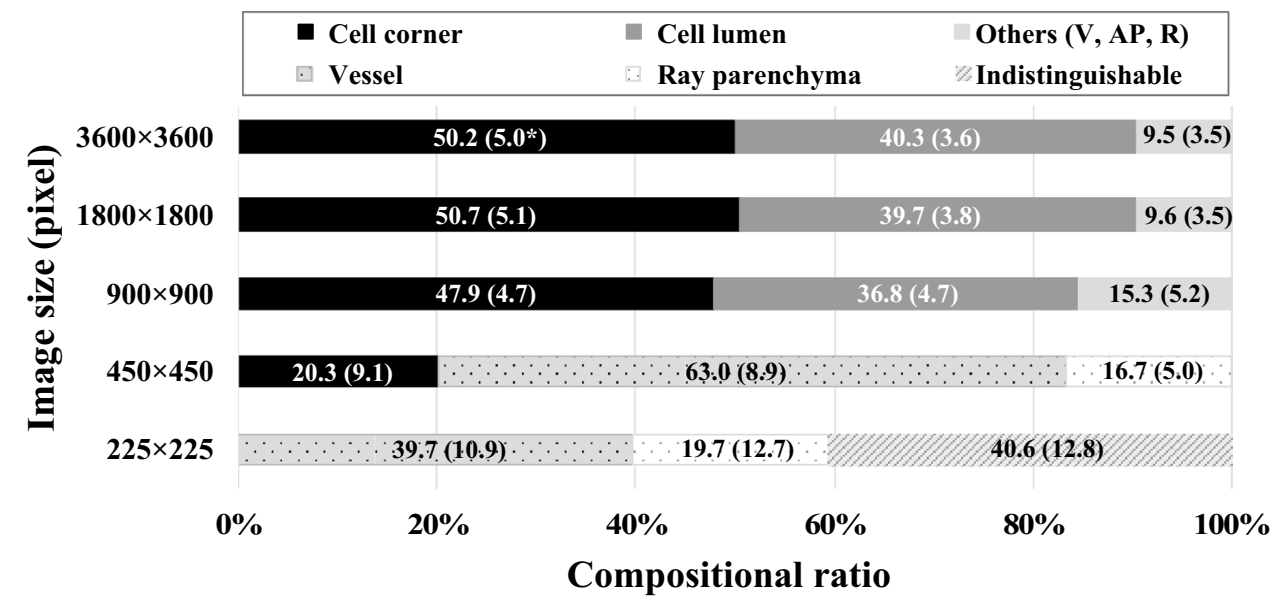


Fig. 7 Dendrograms calculated by the Euclidean distance with Ward's clustering method. Dendrogram at the a genus level and b species level. In the figure, $*$ is the cluster size; $* *$ is the dominant species in the cluster (number of images of the species in the cluster/total number of images of the species in the dataset), and $* * *$ is the minor species in the cluster
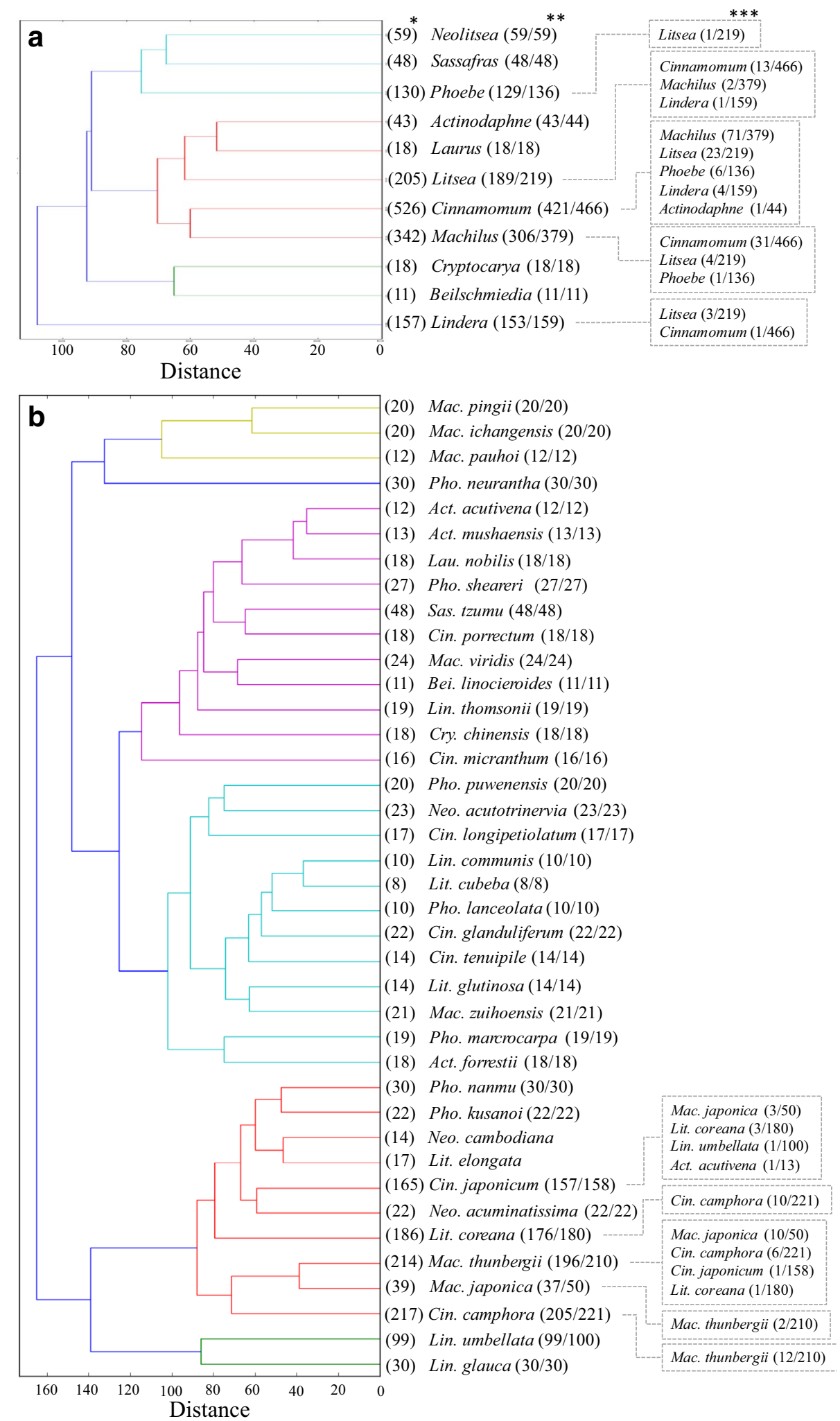

numerical distances of each genus and species are slightly different from the reported phylogenetic relationships [5, 6]. According to Liu et al. [5], who studied the phylogenic relationships of 409 individuals representing 133 species of 12 genera in the Lauraceae using DNA barcoding, 44 individuals from the ten species were misclassified by the form taxonomists. This supports the fact that the morphological features of the Lauraceae are indeed complex to be 
classified unambiguously. As shown in Table 2 and Fig. 3, the fact that the numerical classification by SIFT is more accurate at the species level than at the genus level, implies that the structural features of the species in a given genus are similar to those in another genus, making the genus level classification difficult. This accounts for what the anatomists say about the Lauraceae.

\section{Conclusion}

Feature extraction by SIFT followed by LDA as a classifier allowed us to classify accurately 11 genera and 39 species in the Lauraceae. The extracted features are found to be related to the distributions of the vessels, parenchyma cells, fibers, and ray cells, and thus, providing a new perspective on species-specific morphology. Though such an approach is still relatively new in the wood anatomy, the potential of the technique seems promising, as the computer vision allows us to observe cell corners as a feature which so far has not been considered as species-specific. Ultimately, deep learning by a convoluted neural network may achieve complete automated species identification in reality. Finally, the quantification of two-dimensional image data by computer vision would also allow us to predict the physical and mechanical features of a given wood, if we design the features to reflect the porosity, anisotropy, and ratios of cell types for instance. These lines of investigation are in progress and will be reported elsewhere.

Acknowledgements The authors thank Professor Hiroaki Kawashima, Department of Intelligence Science and Technology, Graduate School of Informatics, Kyoto University, for his useful advice throughout this study. The study was supported by a Grants-in-Aid for Scientific Research (Grant Number 25252033) from the Japan Society for the Promotion of Science, RISH Cooperative Research (database), and RISH Mission Research V to JS, and partly by Natural Science Foundation of Jiangsu Province, China (BK20140981) to ZS. Most of the micrograph database was prepared by Ms. Keiko Kanai and Ms. Izumi Kanai, which is also acknowledged.

Open Access This article is distributed under the terms of the Creative Commons Attribution 4.0 International License (http://creativecommons.org/licenses/by/4.0/), which permits unrestricted use, distribution, and reproduction in any medium, provided you give appropriate credit to the original author(s) and the source, provide a link to the Creative Commons license, and indicate if changes were made.

\section{References}

1. Christenhusz MJM, Byng JW (2016) The number of known plants species in the world and its annual increase. Phytotaxa 261(3):201-217
2. Watanabe U, Abe H, Yoshida K, Sugiyama J (2015) Quantitative evaluation of properties of residual DNA in Cryptomeria japonica wood. J Wood Sci 61(1):1-9

3. Abe H, Watanabe U, Yoshida K, Kuroda K, Zhang C (2011) Changes in organelle and DNA quality, quantity, and distribution in the wood of Cryptomeria japonica over long-term storage. IAWA J 32:263-272

4. Ohyama M, Baba K, Itoh T (2001) Wood identification of Japanese Cyclobalanopsis species (Fagaceae) based on DNA polymorphism of the intergenic spacer between $\operatorname{trn} \mathrm{T}$ and $\operatorname{trn} \mathrm{L} 5^{\prime}$ exon. J Wood Sci 47:81-86

5. Liu ZF, Ci XQ, Li L, Li HW, Conran JG, Li J (2017) DNA barcoding evaluation and implications for phylogenetic relationships in Lauraceae from China. PloS ONE 12(4), e0175788

6. Chanderbali AS, van der Werff HS, Renner S (2001) Phylogeny and historical biogeography of Lauraceae: evidence from the chloroplast and nuclear genomes. Ann Missouri Bot Gard, pp 104-134

7. Muñiz GIB, Carneiro ME, Batista FRR, Schardosin FZ, Nisgoski S (2016) Wood and charcoal identification of five species from the miscellaneous group known in Brazil as "Angelim" by Near-IR and wood anatomy. Maderas Cienc Tecnol 18(3):505-522

8. Hwang SW, Horikawa Y, Lee WH, Sugiyama J (2016) Identification of Pinus species related to historic architecture in Korea using NIR chemometric approaches. J Wood Sci 62:156-167

9. Horikawa Y, Mizuno-Tazuru S, Sugiyama J (2015) Near-infrared spectroscopy as a potential method for identification of anatomically similar Japanese diploxylons. J Wood Sci 61:251-261

10. Hwang SW, Lee WH, Horikawa Y, Sugiyama J (2015) Chemometrics approach for species identification of Pinus densiflora Sieb. et Zucc. and Pinus densiflora for. erecta Uyeki (in Korean). J Korean Wood Sci Technol 43(6):701-713

11. Tsuchikawa S, Inoue K, Noma J, Hayashi K (2003) Application of near infrared spectroscopy to wood discrimination. J Wood Sci 49:29-35

12. Brunner M, Eugster R, Trenka E, Bergamin-Strotz L (1996) FT-NIR spectroscopy and wood identification. Holzforsch 50:130-134

13. Kobayashi K, Hwang SW, Lee WH, Sugiyama J (2017) Texture analysis of stereograms of diffuse-porous hardwood: identification of wood species used in Tripitaka Koreana. J Wood Sci 63:322-330

14. Kobayashi K, Akada M, Torigoe T, Imazu S, Sugiyama J (2015) Automated recognition of wood used in traditional Japanese sculptures by texture analysis of their low-resolution computed tomography data. J Wood Sci 61:630-640

15. Yadav AR, Anand RS, Dewal ML, Gupta S (2015) Multiresolution local binary pattern variants based texture feature extraction techniques for efficient classification of microscopic images of hardwood species. Appl Soft Comput 32:101-112

16. Martins J, Oliveira LS, Nisgoski S, Sabourin R (2013) A database for automatic classification of forest species. Mach Vis Appl 24(3):567-578

17. Wang HJ, Zhang GQ, Qi HN (2013) Wood recognition using image texture features. PLoS ONE 8(10):e76101

18. Viola P, Jones M (2001) Rapid object detection using a boosted cascade of simple features. In: Proceedings of the 2001 IEEE Computer Society Conference on Computer Vision and Pattern Recognition (CVPR). IEEE, Kauai, pp 511-518

19. Dalal N, Triggs B (2005) Histograms of oriented gradients for human detection. In: Proceedings of the 2005 IEEE Computer Society Conference on Computer Vision and Pattern Recognition (CVPR). IEEE, San Diego, pp 886-893 
20. Lowe DG (1999) Object recognition from local scale-invariant features. In: Proceedings of the Seventh IEEE International Conference on Computer Vision. IEEE, Kerkyra, pp 1150-1157

21. Lowe DG (2004) Distinctive image features from scale-invariant keypoints. Intl J Comput Vis 60(2):91-110

22. Huang S, Cai C, Zhang Y (2009) Wood image retrieval using SIFT descriptor. In: International Conference on Computational Intelligence and Software Engineering (CiSE 2009). IEEE, Wuhan, pp 1-4

23. Yang Y, Newsam S (2008) Comparing SIFT descriptors and Gabor texture features for classification of remote sensed imagery. In: 15th IEEE International Conference on Image Processing (ICIP 2008). IEEE, San Diego, pp 1852-1855

24. Mikolajczyk K, Schmid C (2005) A performance evaluation of local descriptors. IEEE Trans Pattern Anal Mach Intell 27(10):1615-1630

25. Bay H, Ess A, Tuytelaars T, Van Gool L (2008) Speeded-up robust features (SURF). Comput Vis Image Underst 110(3):346-359

26. Ke Y, Sukthankar R (2004) PCA-SIFT: A more distinctive representation for local image descriptors. In: Proceedings of the 2004 IEEE Computer Society Conference on Computer Vision and Pattern Recognition (CVPR). IEEE, Washington, pp 506-513
27. Ward JH Jr (1963) Hierarchical grouping to optimize an objective function. J Am Stat Assoc 58:236-244

28. Sokal RR, Rohlf FJ (1962) The comparison of dendrograms by objective methods. Taxon 11:33-40

29. Python Software Foundation (2016) Python version 3.5 series. https://www.python.org/. Accessed 07 Nov 2016

30. Itseez (2016) Open source computer vision library. http://opencv. org/. Accessed 07 Nov 2016

31. Van der Walt S, Colbert SC, Varoquaux G (2011) The NumPy array: a structure for efficient numerical computation. Comput Sci Eng 13(2):22-30

32. Jones E, Oliphant T, Peterson P (2014) SciPy: Open source scientific tools for Python. http://www.scipy.org/. Accessed 07 Nov 2016

33. Pedregosa F, Varoquaux G, Gramfort A, Michel V, Thirion B, Grisel O, Blondel M, Prettenhofer P, Weiss R, Dubourg V, Vanderplas J, Passos A, Cournapeau D, Brucher M, Perrot M, Duchesnay É (2011) Scikit-learn: Machine learning in Python. J Mach Learn Res 12:2825-2830 